\title{
EHD friction properties of ISO VG 320 gear oils with smooth and rough surfaces
}

\author{
Balasubramaniam VENGUDUSAMY*, Claus ENEKES, Reiner SPALLEK \\ Klüber Lubrication München SE \& Co. KG, Geisenhausenerstraße 7, Munich 81379, Germany \\ Received: 20 April 2018 / Revised: 22 Ocotober 2018 / Accepted: 23 December 2018 \\ (C) The author(s) 2019.
}

\begin{abstract}
The elastohydrodynamic (EHD) friction properties of seven ISO VG 320 gear oils including three polyalphaolefins (PAOs), three polyglycols (PG) and a mineral oil have been investigated in rolling/sliding conditions at six different temperatures and three roughnesses. Film thickness, Stribeck and traction curves have been generated using a ball-on-disc tribometer. Film thickness results are in agreement with previous work that it is primarily controlled by pressure-viscosity coefficient and viscosity of lubricants. The results with smooth surface show that all oils experience significant shear heating leading to friction reduction at higher strain rates or lambda ratios but only PGs reach limiting friction whereas mineral oil and PAOs do not. Friction curves obtained at different temperatures and roughnesses enable simulating an extensive range of lubrication regimes and allow isothermal friction correction for shear heating. Stribeck curves with rough surfaces show an increase in friction in the lambda range of $0.5-3.5$, where asperity separation varies from partial to full-indicating that roughness effects can be expected even under full film condition. This increase in friction is attributed to formation of a micro-EHD region, and is seen only with mineral oil and PAOs whereas not with PGs. The results also highlight how EHD friction properties of different family of fluids could be influenced by roughness effects, and the possible mechanisms are discussed.
\end{abstract}

Keywords: ISO VG 320 gear oil; roughness effect; film thickness; friction; EHD; micro-EHD

\section{Introduction}

Many engineering components like rolling bearings, gears and cams operate in elastohydrodynamic (EHD) lubrication condition. Increasing the efficiency of these components is becoming an ever-increasing challenge as this helps reduce greenhouse gas emissions to a great extent. The efficiency can generally be improved either by using optimized surfaces (e.g. surfaces with lower surface roughness, coatings) and/or by reducing frictional losses. The latter can be achieved by use of lubricants with low viscosity, low pressure-viscosity coefficient and low shear strength [1,2]. This drives the current trend towards the use of less viscous lubricants [3-5]. However, the downside associated with the use of such lubricants is the formation of thinner lubricant films, leading to mixed lubrication and early wear. The problem of wear is generally addressed by use of functional additives that provide adequate surface protection. But the friction in mixed lubrication is generally higher than that in EHL (elastohydrodynamic lubrication) and is known to be influenced by roughness and lubricant properties. So, the idea of using less viscous lubricants may have some compromises on the expected energy savings when roughness effects are not analysed. It is therefore important to gain a better understanding of the effects of roughness on friction in mixed lubrication.

Although several previous studies have been carried out on EHD friction [3, 6-13], there is still considerable debate as to the origins of EHD friction [14], especially for lubricated rough contacts. Experimental studies have

* Corresponding author: Balasubramaniam VENGUDUSAMY, E-mail: Balasubramaniam.Vengudusamy@klueber.com 
proven that EHD friction is controlled by molecular structure [15-18] and rheology of lubricants [19, 20]. Currently, many rheological models exist to describe the friction behavior of lubricants in EHD contact. One such is Carreau model. Generally, the friction behaviour of lubricants in the form of traction curves can follow one of the patterns shown schematically in Fig. 1. Type I is the well-known Newtonian behaviour where the shear stress increases linearly with the log (shear rate) whereas with types II \& III lubricants behave Newtonian only at low slide-roll ratios (SRR) or shear rates and non-Newtonian at high shear rates. It is widely accepted that friction increases rapidly at low SRR and either level out (type II) or reach a limiting stress, level out and drop at high SRRs (type III). The drop in friction at high shear rates is generally attributed to shear heating of the EHD film [11]. It is however unclear whether some fluids (type III) really reach the limiting friction or whether the temperature rise of the oil film was much higher for the friction to drop even before the limiting friction was reached. This can be studied by obtaining isothermal friction curves [18]. The shapes of traction curves shown in Fig. 1 are mainly based on results from smooth surfaces. Much less work exists on the shapes of traction curves for rough surfaces.

In general, studying the effects of surface parameters on film thickness and friction is practically very important as they simulate features or effects similar to indents that are commonly seen in bearing raceways and gears. In this context, some previous studies have reported an increase in friction with rough surfaces

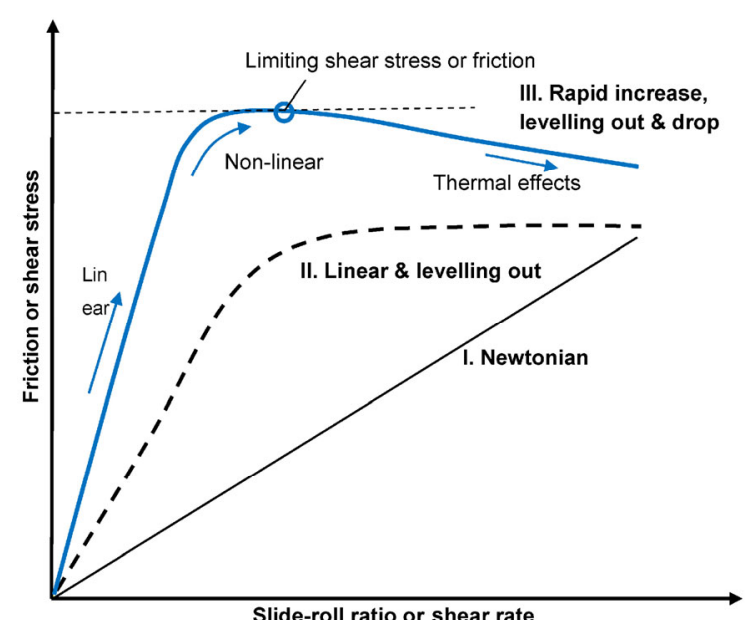

Fig. 1 Schematic diagram showing different patterns of traction curves [14]. where a micro-EHD film is suggested to form $[3,6,7$, 21]. The increase in friction is believed to be due to higher pressure and thinner films at the asperity peaks causing a local increase in lubricant viscosity and shear stress [3]. Nanbu et al. [22] studied the effect of surface roughness on EHD friction and reported an increase in shear stress and attributed this to viscosity increase due to pressure fluctuations. A study by Björling et al. [23] on circumferential rough surfaces ( $S_{a}: 80 \mathrm{~nm}$ and $220 \mathrm{~nm}$ disc) also showed a higher friction with the rough surfaces but they attributed this to formation of an earlier mixed lubrication. Another study by Zapletal et al. [24] on uniform surface textures revealed that friction increase could happen even before any direct contact between surfaces occurred. They attributed this to the viscosity change due to pressure ripples caused by surface roughness. Although the surfaces they studied were not rough enough $\left(R_{q}\right.$ : between $2 \mathrm{~nm}$ and $15 \mathrm{~nm}$ ), they still observed the roughness effects on friction. This tends to suggest that effect of pressure caused by roughness on lubricant viscosity is much more significant compared to the absolute roughness value. It is also believed that surface roughness parameters like $R_{a}$ and $R_{q}$ alone may not completely represent the surface, hence effects of other parameters like waviness and wavelength should also be considered to understand their effects on friction and film thickness. Venner and Lubrecht $[25,26]$ studied this with a sinusoidal waviness surface represented by wavelength and undeformed amplitude. They reported that rough surfaces in EHD contact encounter more deformation than outside and this depends on the wavelength. Surfaces with long wavelength were reported to deform completely while no changes with those with short wavelength. However, a study by Guegan et al. [27] showed that wavelength does not affect film formation much compared to the effects imparted by RMS (root mean square) roughness. They also reported the formation of micro-EHL film at the asperity ridges. Another study by Greenwood and Morales-Espejel [28] reported similar to Venner and Lubrecht $[25,26]$ that asperity flattening is significant when they enter the EHD contact. They also highlighted the significance of asperity deformation by studying a transverse roughness pattern and showed that film formation will differ depending on whether a valley or peak enters the EHD contact. For instance, a valley 
will entrain large amount of lubricant compared to an asperity, resulting in a fluctuating entrainment and film formation.

Furthermore, one limitation of most previous work is that these studies have tended to focus mainly on a particular base fluid. Also since the conditions that gear contacts encounter are much severe (rough surface, high slide-roll ratio, medium to high temperature, high contact pressure, medium to high lambda ratio), it is of great importance to understand how gear oils behave with rough surfaces in mixed lubrication. Such conditions, for instance, prevail very much on wind turbine gearboxes. In light of all these, this paper examines the EHD friction properties of seven gear oils including a mineral oil, three PAOs and three PGs with three roughnesses and six temperatures in a rolling/sliding contact. This study also explores the effects of roughness on mixed/EHD friction and whether the effects are similar for all base fluids.

\section{Test methods}

\subsection{Friction}

The friction tests were carried out using a ball-on-disc EHD rig (PCS Instruments), where a steel ball half submerged in lubricant was loaded and rubbed in rolling/sliding conditions against a steel disc. A typical test included two steps namely, generating Stribeck and traction curves. Stribeck curves were obtained by measuring friction while varying entrainment speeds in stages from $0.004 \mathrm{~m} / \mathrm{s}$ up to $3.2 \mathrm{~m} / \mathrm{s}$ at a fixed slide-roll ratio of $50 \%$. Traction curves were obtained by measuring friction at a constant entrainment speed of $2.5 \mathrm{~m} / \mathrm{s}$ while varying the SRR from 0 to $100 \%$. Tests were carried out at six temperatures, $25^{\circ} \mathrm{C}, 40{ }^{\circ} \mathrm{C}, 50{ }^{\circ} \mathrm{C}$, $60{ }^{\circ} \mathrm{C}, 80^{\circ} \mathrm{C}$ and $100{ }^{\circ} \mathrm{C}$, and $36 \mathrm{~N}$, corresponding to a maximum Hertz contact pressure of $1 \mathrm{GPa}$. The entrainment speed was defined as $\left(u_{\mathrm{b}}+u_{\mathrm{d}}\right) / 2$, where $u_{\mathrm{b}}$ and $u_{\mathrm{d}}$ respectively are the speed of the ball and disc with respect to the contacting surfaces, while the SRR was defined as the ratio of sliding speed $\left|u_{\mathrm{b}}-u_{\mathrm{d}}\right|$ to entrainment speed.

The ball and disc specimens used were made of AISI 52100 steel, had hardness of $600 \mathrm{HV}$ and of diameters $19 \mathrm{~mm}$ and $100 \mathrm{~mm}$, respectively. Three disc roughnesses $R_{a}$ of $0.02 \mu \mathrm{m}, 0.2 \mu \mathrm{m}$ and $0.35 \mu \mathrm{m}$ corresponding respectively to $R_{q}$ of $0.025 \mu \mathrm{m}, 0.22 \mu \mathrm{m}$ and $0.38 \mu \mathrm{m}$, and $R_{k u}$ of $2.465,3.147$ and 3.526 were studied in this work. The specimens with lowest roughness $\left(R_{a}=0.02 \mu \mathrm{m}\right)$ and those with higher roughness $\left(R_{a}=0.2 \mu \mathrm{m}\right.$ and $\left.0.35 \mu \mathrm{m}\right)$ will hereafter be referred to as smooth and rough surfaces, respectively. The roughness of rough surfaces was achieved by lapping, hence no specific orientation of asperities. The $R_{k u}>3$ of rough specimens indicate that the two rough surfaces investigated had sharp peaks and valleys (Fig. S1 in the Electronic Supplementary Material (EMS)) compared to the smooth surface. The counterface balls were in all cases very smooth, of roughness $R_{a}=10 \mathrm{~nm}$. Fresh specimens were used for each test lubricant. This means that the test protocol included obtaining Stribeck and tractions curves at several temperatures indicated above, all using the same specimen. Each Stribeck and traction curve lasted for about $5 \mathrm{~min}$; so totally $60 \mathrm{~min}$ for 6 test temperatures (both Stribeck \& traction curves) plus the heating time. Since boundary lubrication region in each Stribeck curve step was roughly only for a period of $1 \mathrm{~min}$, change in surface roughness is unlikely. Some surface roughness measurements made after the test showed that roughness did not change much as expected. This was important for two reasons, one, to attribute the observed friction effects solely to lubricants and two, to base all lambda calculations on the unworn surfaces. It should be noted that tests were repeated twice and the deviation in friction between two tests was less than $10 \%$. Some representative curves from repeat tests are shown in Fig. S2 in the EMS, where it can be seen that curves from two tests overlap well.

\subsection{Film thickness}

The film thickness was measured using the same EHD rig used for friction measurements but with a transparent glass disc instead of the steel disc. This setup works on optical interferometry principles by combining spacer layer and spectrometry to measure central film thickness in a rolling or rolling/sliding point contact formed between a steel ball and a glass disc [29]. The central film thickness, $h_{\mathrm{c}}$ was measured at the same six temperatures used in friction tests under pure rolling condition for an applied load of $50 \mathrm{~N}$. However, only the results of extreme temperature 
cases $\left(40{ }^{\circ} \mathrm{C}, 80{ }^{\circ} \mathrm{C}\right.$ and $\left.100{ }^{\circ} \mathrm{C}\right)$ are shown here as representative measurements. The film thickness measurements were made firstly to understand the film forming properties and secondly to enable shear rate calculation. Tests were repeated and their curves from two tests overlapped well, ensuring that the observed phenomena are reliable. In general, deviation in film thickness between two tests was less than $3 \%$.

Both for friction and film thickness tests, the test specimens and rig accessories were ultrasonically cleaned in isopropanol and dried before they were assembled in the rig. The lubricant pot was filled with the test oil so that half the ball was submerged.

\section{Test lubricants}

Seven fully formulated ISO VG 320 gear oils including one mineral oil, three PAOs and three PGs were investigated, and their properties are listed in Table 1. The pressure-viscosity coefficients $(\alpha)$ were determined by comparing EHD film thickness of a test lubricant with that of a reference oil of known $\alpha$-value [30]. Only data at speeds above $0.1 \mathrm{~m} / \mathrm{s}$ were used for $\alpha$-estimation. The chosen lubricants were all of similar viscosity at $40{ }^{\circ} \mathrm{C}$ but differ by base oil type and additivation. These lubricants contain P-S-based EP/AW additives but no viscosity modifiers. Since the test protocol did not include any significant boundary lubrication, the additives have very negligible effect on the measurements. The different temperatures and roughnesses chosen in this study were to allow different lubrication regimes and conditions to be studied,

Table 1 Gear oil properties.

\begin{tabular}{|c|c|c|c|c|c|c|}
\hline \multirow[t]{2}{*}{ No. } & \multirow[t]{2}{*}{$\begin{array}{l}\text { Base oil } \\
\text { type \& Oil } \\
\text { code }\end{array}$} & \multicolumn{2}{|c|}{$\begin{array}{l}\text { Kinematic } \\
\text { viscosity } \\
\left(\mathrm{mm}^{2} / \mathrm{s}\right)\end{array}$} & \multirow[t]{2}{*}{$\begin{array}{l}\text { Viscosity } \\
\text { index }\end{array}$} & \multicolumn{2}{|c|}{$\begin{array}{l}\text { Pressure-viscosity } \\
\text { coefficient } \alpha \\
\left(\mathrm{GPa}^{-1}\right)^{*}\end{array}$} \\
\hline & & $40{ }^{\circ} \mathrm{C}$ & $100{ }^{\circ} \mathrm{C}$ & & $40{ }^{\circ} \mathrm{C}$ & $100{ }^{\circ} \mathrm{C}$ \\
\hline 1 & $\begin{array}{l}\text { Mineral } \\
\text { (Min) }\end{array}$ & 320 & 25 & 90 & 26 & 10.7 \\
\hline 2 & PAO 1 & 320 & 35 & 145 & 13 & 9.0 \\
\hline 3 & PAO 2 & 320 & 40 & 175 & 17 & 7.5 \\
\hline 4 & PAO 3 & 320 & 35 & 160 & 16 & 9.7 \\
\hline 5 & PG 1 & 320 & 55 & 230 & 10 & 6.4 \\
\hline 6 & PG 2 & 320 & 55 & 230 & 12 & 7.0 \\
\hline 7 & PG 3 & 320 & 55 & 230 & 12 & 6.8 \\
\hline
\end{tabular}

including shear heating and roughness effects on EHD friction and micro-EHD film formation. Testing ISO VG 320 oils at different temperatures allows varying the viscosity, enabling to study other ISO VG grades as well. For example, the mineral oil used in this study is ISO VG 320, 220, 100 and 46 at $40{ }^{\circ} \mathrm{C}, 50{ }^{\circ} \mathrm{C}, 60{ }^{\circ} \mathrm{C}$ and $80^{\circ} \mathrm{C}$, respectively. Since in some cases bearings in gearboxes are also lubricated by gear oils, the results from this work can be practically relevant for both bearings and gears although however their roughness patterns differ.

\section{Results}

\subsection{Film thickness}

The film thickness measurements made as a function of speed for seven gear oils at three temperatures are shown in Fig. 2. In all cases, film thickness increases
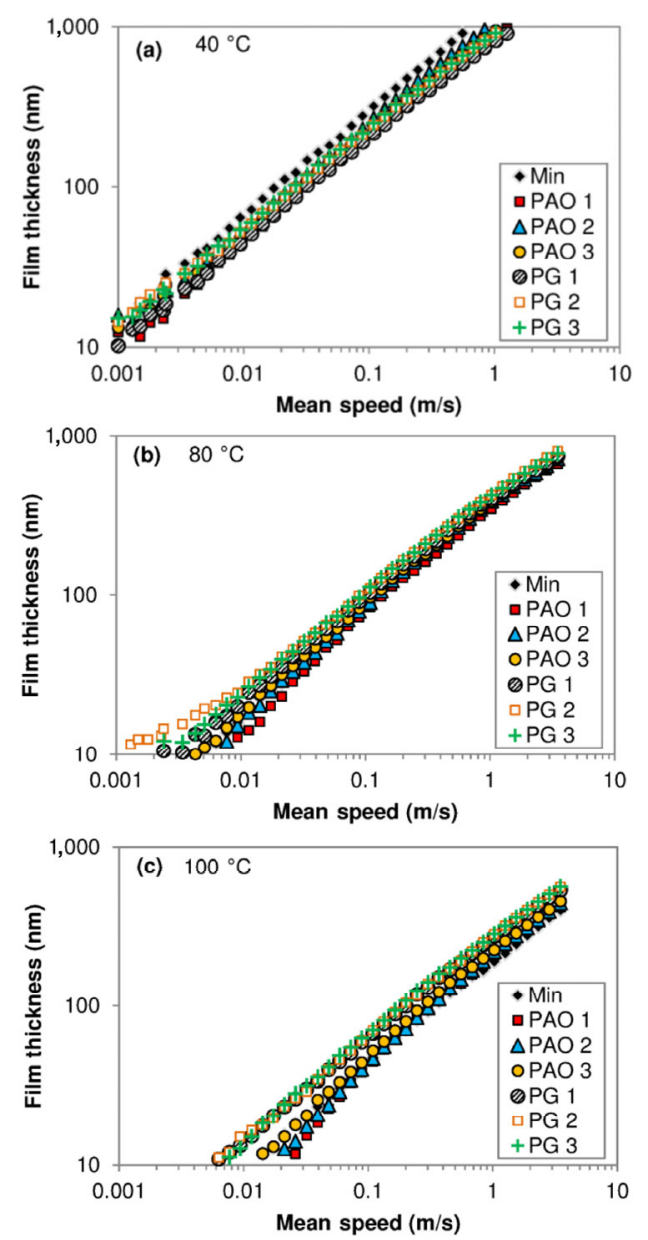

Fig. 2 Film thickness as a function of mean speed for (a) $40{ }^{\circ} \mathrm{C}$, (b) $80{ }^{\circ} \mathrm{C}$, and (c) $100{ }^{\circ} \mathrm{C}$. 
with speed, following the classical EHL theory with $\log$-log film thickness-speed exponent 0.67 . It is evident by comparing film thickness at high speeds $(2.5 \mathrm{~m} / \mathrm{s})$ that film thickness is controlled by $\alpha$ at low temperature (Fig. 3(a)) while by VI (or viscosity) at high temperature (Fig. 3(b)). Since all oils have similar viscosity $\left(320 \mathrm{~mm}^{2} / \mathrm{s}\right)$ at $40{ }^{\circ} \mathrm{C}$, film thickness is primarily controlled by their $\alpha$-value, thus mineral oil forms a thicker film followed by PAOs and PGs. The film thickness of PGs at $40{ }^{\circ} \mathrm{C}$ does not vary much due to their similar $\alpha$-value. However, the above-said correlation between film thickness and $\alpha$ is not seen at $100{ }^{\circ} \mathrm{C}$ because of the difference in viscosity. Mineral oil has lower viscosity than PAOs and PGs (see Table 1). So, film thickness at $100{ }^{\circ} \mathrm{C}$ is dominated by viscosity instead of $\alpha$ (Fig. 3(c)).
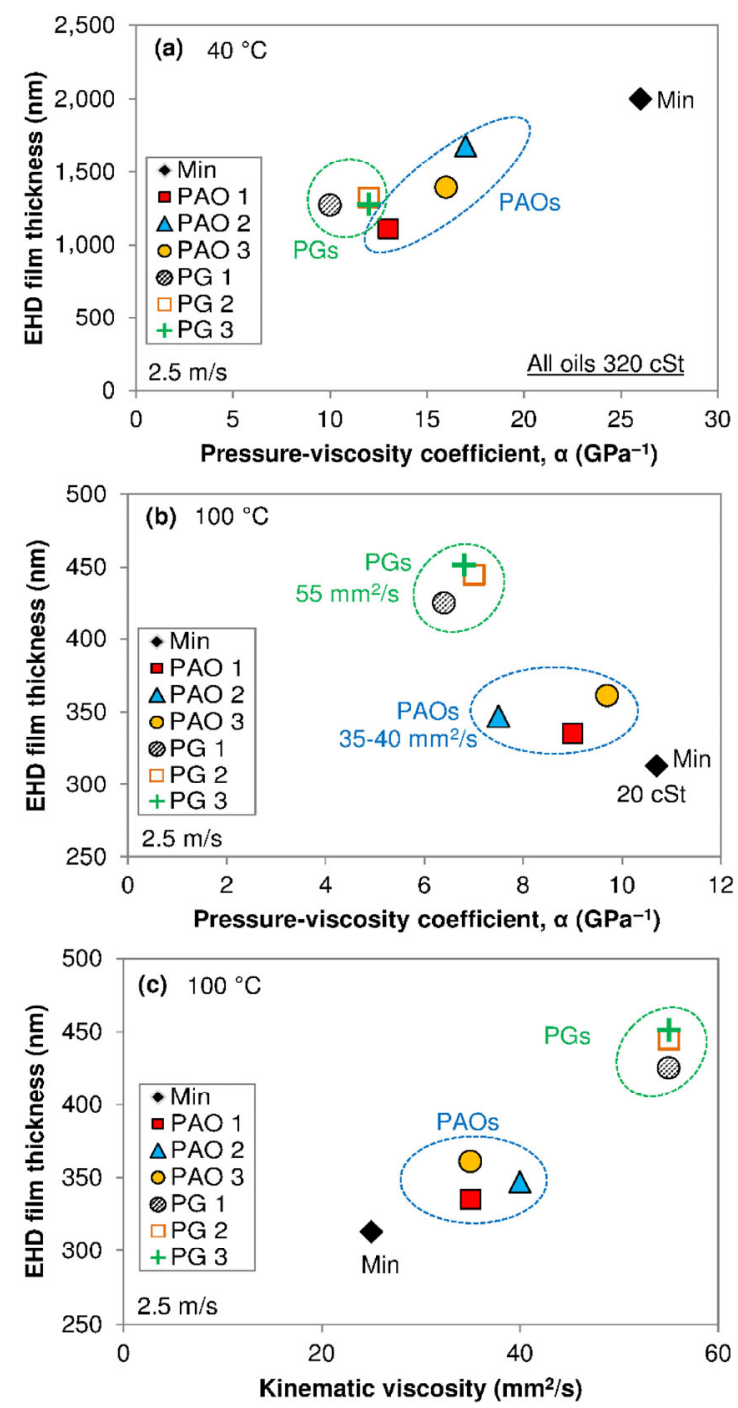

Fig. 3 EHD film thickness at $2.5 \mathrm{~m} / \mathrm{s}$ (a) versus $\alpha$ for $40{ }^{\circ} \mathrm{C}$, (b) versus $\alpha$ for $100{ }^{\circ} \mathrm{C}$, and (c) versus kinematic viscosity for $100{ }^{\circ} \mathrm{C}$.
It should however be noted that the observed effects may not be a general conclusion, but rather the effect of the test lubricants being similar in viscosity at $40^{\circ} \mathrm{C}$. If the lubricants were chosen to have similar viscosities at $100{ }^{\circ} \mathrm{C}$, the film thickness at that temperature would be dominated by pressure-viscosity coefficient and probably by viscosity at $40{ }^{\circ} \mathrm{C}$.

\subsection{Friction}

The friction coefficient versus entrainment speed curves (Stribeck curves) obtained at different temperatures and roughnesses for one of the gear oils (Min) are shown in Fig. 4. The measured EHD film thickness values shown in Fig. 3 were used to predict the film thickness and lambda ratio $(\lambda$, ratio of film thickness
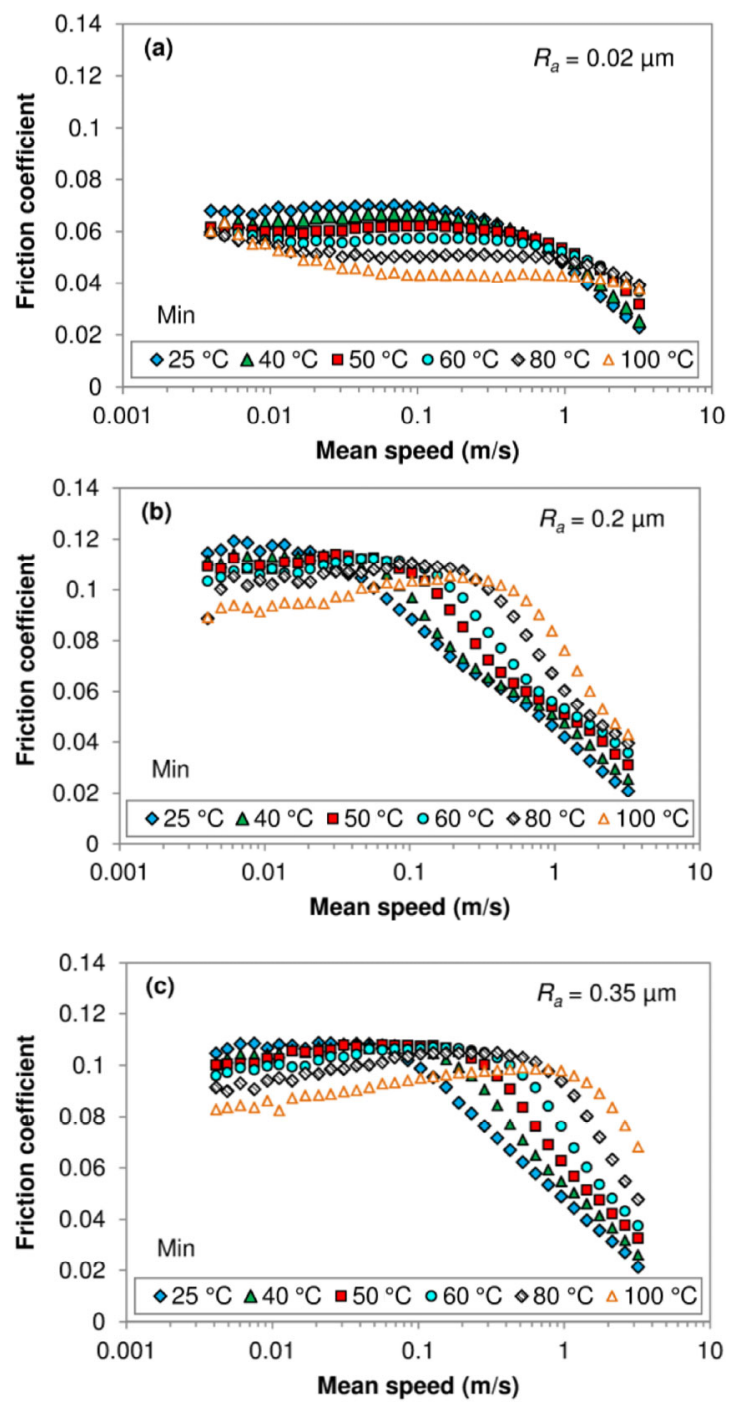

Fig. 4 Stribeck curves measured for mineral oil with (a) $R_{a}=$ $0.02 \mu \mathrm{m}$, (b) $R_{a}=0.2 \mu \mathrm{m}$, and (c) $R_{a}=0.35 \mu \mathrm{m}$ 
to composite roughness $R_{q}$ ) for smooth surface in friction tests by taking into account the difference in material (steel) and load using the Dowson EHD point contact equation [31]. This adjustment is similar to that reported in Ref. [18] and the factor was 0.97 . However, it should be noted that the effect of increased sliding in friction tests on film thickness was not taken into account while making the film thickness adjustment. Furthermore, the film thickness with rough surfaces would normally be less compared to that formed with a smoother one [27]. So lambda ratios for rough surfaces were obtained by adjusting the lambda ratio of smooth surface by a factor. The terms lambda ratio and modified lambda ratio used throughout the article correspond to smooth and rough surfaces, respectively.

The required factors for the two roughnesses studied were obtained by a 3-step procedure described in Fig. S3 shown in the ESM. For this, film thickness results reported by Guegan et al. [27] for different roughnesses were used, as illustrated in step 1 in Fig. S3 in the ESM. First four columns in the table shown in step 1 list the film thickness data (values not shown instead only the method is illustrated) generated by [27] for $R_{a}=0.15 \mu \mathrm{m}$ and $0.27 \mu \mathrm{m}$ at different speeds. Columns 5 and 6 in the table shows the film thickness ratio obtained between rough and smooth surfaces, i.e. $h_{\text {rough }} / h_{\text {smooth }}$ and $h_{\text {rough } 2} / h_{\text {smooth, }}$ respectively for the two roughnesses [27] used. The obtained ratio for the whole speed range is then averaged for each roughness (referred to as average film thickness ratio, $h_{\text {rough }} / h_{\text {smooth }}$ ), shown as A and B for roughness 1 and 2, respectively in step 2.The data obtained in step 2 (i.e. average film thickness ratio and roughness) were then smooth fitted by $2^{\text {nd }}$ order polynomial equation, as illustrated in step 3. By using the fitted equation, the required film thickness ratios for the roughnesses used in this study were extrapolated. The obtained ratios or factors were $h_{\text {rough }\left(R_{a}=0.2 \mu \mathrm{m}\right)} / h_{\text {smooth }\left(R_{a}=0.02 \mu \mathrm{m}\right)}=0.35$ for $R_{a}=0.2 \mu \mathrm{m}$ surface and $h_{\text {rough }\left(R_{a}=0.35 \mu \mathrm{m}\right)} / h_{\text {smooth }\left(R_{a}=0.02 \mu \mathrm{m}\right)}=0.18$ for $R_{a}=$ $0.35 \mu \mathrm{m}$ surface. Since this study used a similar roughness for the smooth surface, contact geometry and test rig as used by Guegan et al., the fitted factors obtained based on their results predict the lambda ratios fairly well for the roughnesses used in this study. It should however be noted that their study used a longitudinal roughness pattern in contrast to the structure used in the present study. According to average flow model the $h_{\text {rough }} / h_{\text {smooth }}$ ratio would approach 1 if lambda is very large, and as the lambda decreases, the ratio could be less or larger than 1 depending on the orientation of surface roughness. Any effects due to the difference in roughness patterns are neglected in the procedure described above and possibly, because of this, Stribeck curves of $R_{a}=0.35 \mu \mathrm{m}$ did not merge well with other roughness curves when plotted against modified lambda ratio as otherwise normally would. The film thickness ratios obtained based on the reported longitudinal roughness appears to predict fairly well the film thickness for the $R_{a}=0.2 \mu \mathrm{m}$ whereas not for $R_{a}=0.35 \mu \mathrm{m}$. For this reason, the film thickness ratio factor for $R_{a}=0.35 \mu \mathrm{m}$ surface was further adjusted by a trial and error method to find the value at which Stribeck curves merged well (see Fig. S4 in the ESM), which was found to be $h_{\mathrm{rough}\left(R_{a}=0.35 \mu \mathrm{m}\right)} / h_{\mathrm{smooth}\left(R_{a}=0.02 \mu \mathrm{m}\right)}=$ 0.3 for $R_{a}=0.35 \mu \mathrm{m}$ surface. It can be seen from Fig. S4 in the ESM that curves of $R_{a}=0.35 \mu \mathrm{m}$ surface shifts towards left side due to their higher roughness and merge well with those of $R_{a}=0.2 \mu \mathrm{m}$ surface in the medium lambda range. The obtained film thickness reduction factors are approximations, intended mainly to emphasize the observed friction effects.

The calculated lambda ratios were plotted against friction coefficients for different temperatures, as shown in Fig. 5. Friction curves of other gear oils are added in Fig. S5 in the ESM. These curves are plotted to judge lubrication regimes, which was based on Stribeck curve shapes (high friction at low speed or low lambda ratio, and low friction at high speed or high lambda ratio, correspond to boundary lubrication, and EHL regime, respectively) and calculated lambda ratio $(\lambda<1$ and $\lambda>1$ correspond to boundary lubrication and EHL regime, respectively). As can be seen from Fig. 5(a), smooth surface with $R_{a}=0.02 \mu$ m encountered EHL regime for the whole speed range at temperatures $25{ }^{\circ} \mathrm{C}-60{ }^{\circ} \mathrm{C}$ while at elevated temperatures showed formation of mixed lubrication at low speeds. The rough surfaces encountered mixed/boundary regime at high temperatures (lambda ratio $<1$ ) and mixed/ boundary + EHL regimes at low temperatures. As the viscosity and film thickness drops due to increase in temperature, curves shift towards left side. This means at low speeds and $100{ }^{\circ} \mathrm{C}$ more mixed lubrication with smooth surface and more boundary lubrication with 
rough surfaces, as shown schematically in Fig. S6 in the ESM. The curve shapes of smooth surface shown in Fig. 5(a) look different compared to that typically known. This is mainly because lubricants of such high viscosity form a very thick film, leading to complete contact separation even at low speeds. Hence, curves show only the tail part (EHL) of the conventionally known Stribeck curve as shown in Fig. S6 in the ESM. Similar shapes have been reported for high viscosity lubricants in the previous works [32].
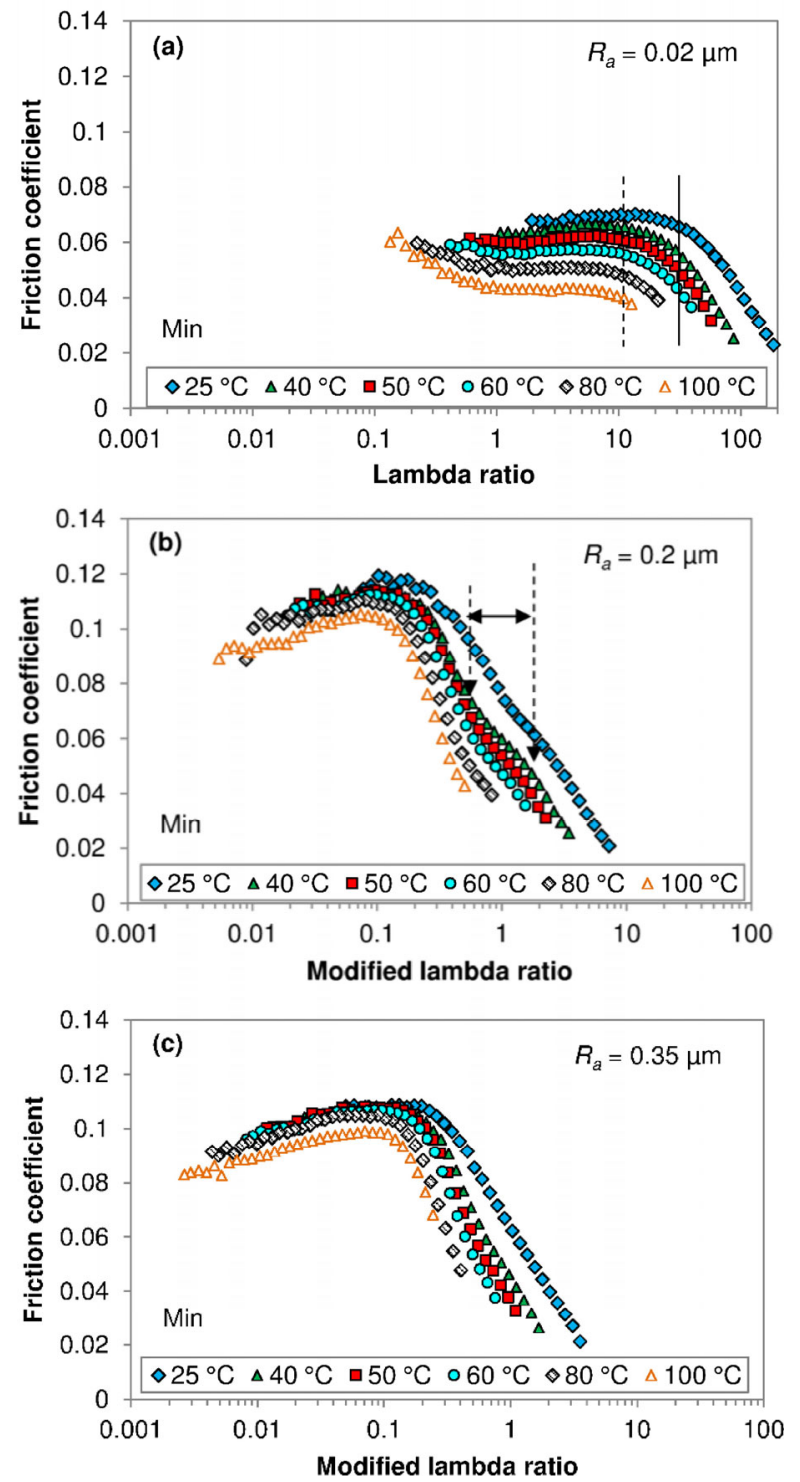

Fig. 5 Friction versus lambda ratio curves measured for mineral oil with (a) $R_{a}=0.02 \mu \mathrm{m}$, (b) $R_{a}=0.2 \mu \mathrm{m}$, and (c) $R_{a}=0.35 \mu \mathrm{m}$. The vertical thick and dashed lines in (a) indicates the lambda ratio beyond which shear heating of EHD film occurs, causing a drop in friction for $25^{\circ} \mathrm{C}$ and $100{ }^{\circ} \mathrm{C}$, respectively. Arrows shown in (b) indicate the region where increase in friction is observed.
Interestingly, all gear oils with smooth surface and low temperatures show a significant reduction in friction at higher lambda ratios (see Fig. 5(a) for mineral oil and Figs. S5(a), S5(d), S5(g), S5(j), S5(m) and S5(p) in the EMS for other gear oils), indicating that shear heating of the EHD film is significant at $\lambda>15$ (indicated by a vertical thick and dashed line in Fig. 5(a) for $25^{\circ} \mathrm{C}$ and $40{ }^{\circ} \mathrm{C}-100{ }^{\circ} \mathrm{C}$, respectively). Shear heating of EHD film results from the temperature rise of the oil film caused by frictional heat dissipated in the contact [18]. However, this was much less pronounced with rough surfaces due to thin film and relatively lower lambda ratios at high speeds. Furthermore, it can be seen that for the same lambda ratio $(\lambda \sim 10)$ friction at $25{ }^{\circ} \mathrm{C}$ with $R_{a}=0.2 \mu \mathrm{m}$ surface (Fig. 5(b)) is lower ( 0.02) while with $R_{a}=0.02 \mu \mathrm{m}$ surface (Fig. 5(a)) is much higher ( 0.07). This could be attributed to complicated difference in their lubrication regimes. For instance, while contacts with $R_{a}=0.02 \mu \mathrm{m}$ show a flat friction region followed by a drop in friction (EHL followed by shear heating), contacts with $R_{a}=0.2 \mu \mathrm{m}$ do not show the flat EHL region at all. Due to this difference in lubrication regimes, their friction levels differ for the same lambda ratio. It is believed that lambda ratio alone may not be sufficient to predict the lubrication regimes properly [24], especially when the difference in roughness is significant. This possibly could also be the reason why curves obtained at different temperatures do not overlap when friction was plotted against lambda ratio (Fig. S5 in the ESM).

Figure 6 shows a typical set of friction versus SRR curves (traction curves) obtained for mineral oil at different temperatures with three roughnesses while Fig. 7 shows traction curves of PAOs and PGs obtained with the smooth surface. Traction curves for other roughnesses are presented in Fig. S7 in the ESM. As can be seen from Figs. 6(a) and 7, friction increases rapidly at low SRR followed by either levelling out or a drop at high SRR, broadly following one or more of the patterns shown in Fig. 1. As widely known, mineral oil shows higher EHD friction followed, in order, by PAOs and PGs as shown in Fig. S7 in the ESM. This is attributed to the difference in their molecular structure and $\alpha$-value. The results are in agreement with the known existing correlation between $\alpha$, VI and EHD friction, i.e. lubricants with high $\alpha$-value and low VI (mineral) show high EHD friction and vice versa as 

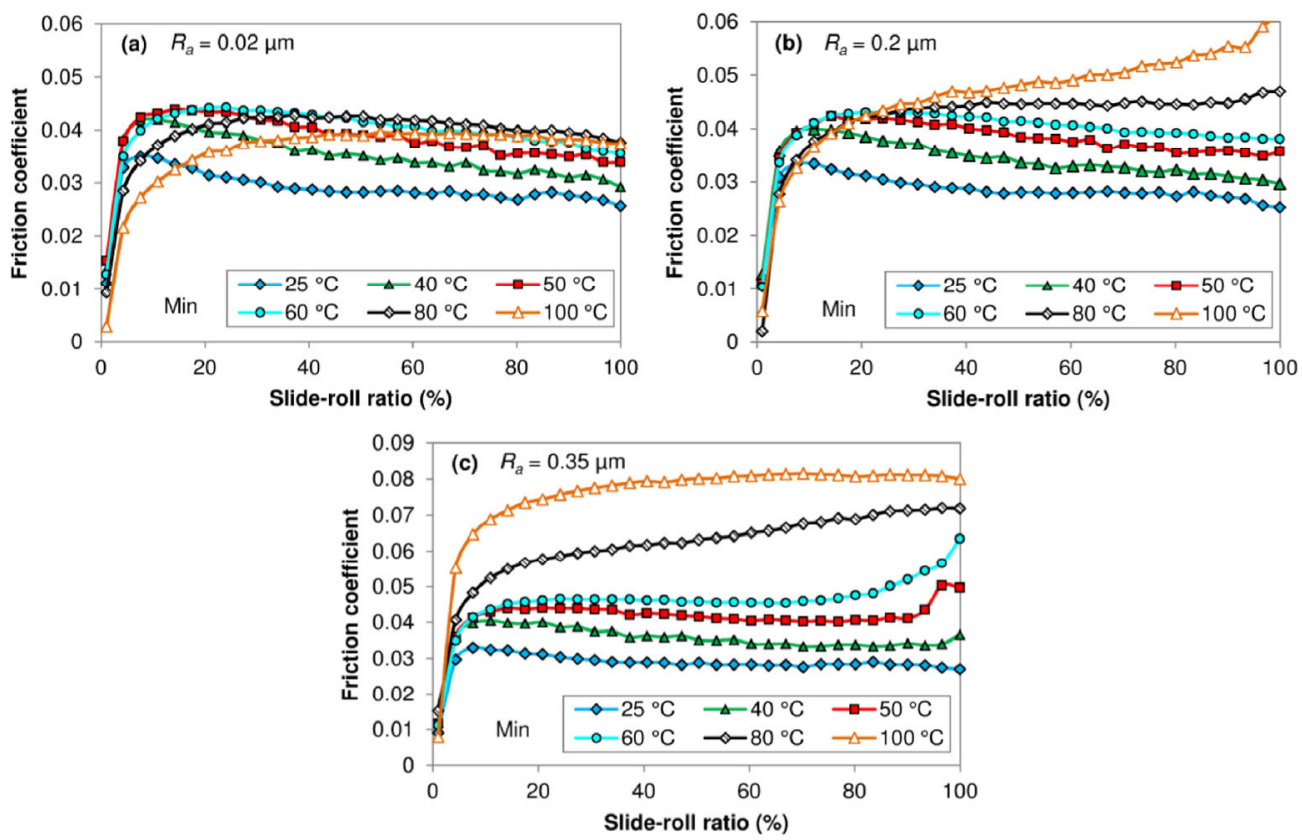

Fig. 6 Traction curves measured for mineral oil with (a) $R_{a}=0.02 \mu \mathrm{m}$, (b) $R_{a}=0.2 \mu \mathrm{m}$, and (c) $R_{a}=0.35 \mu \mathrm{m}$.
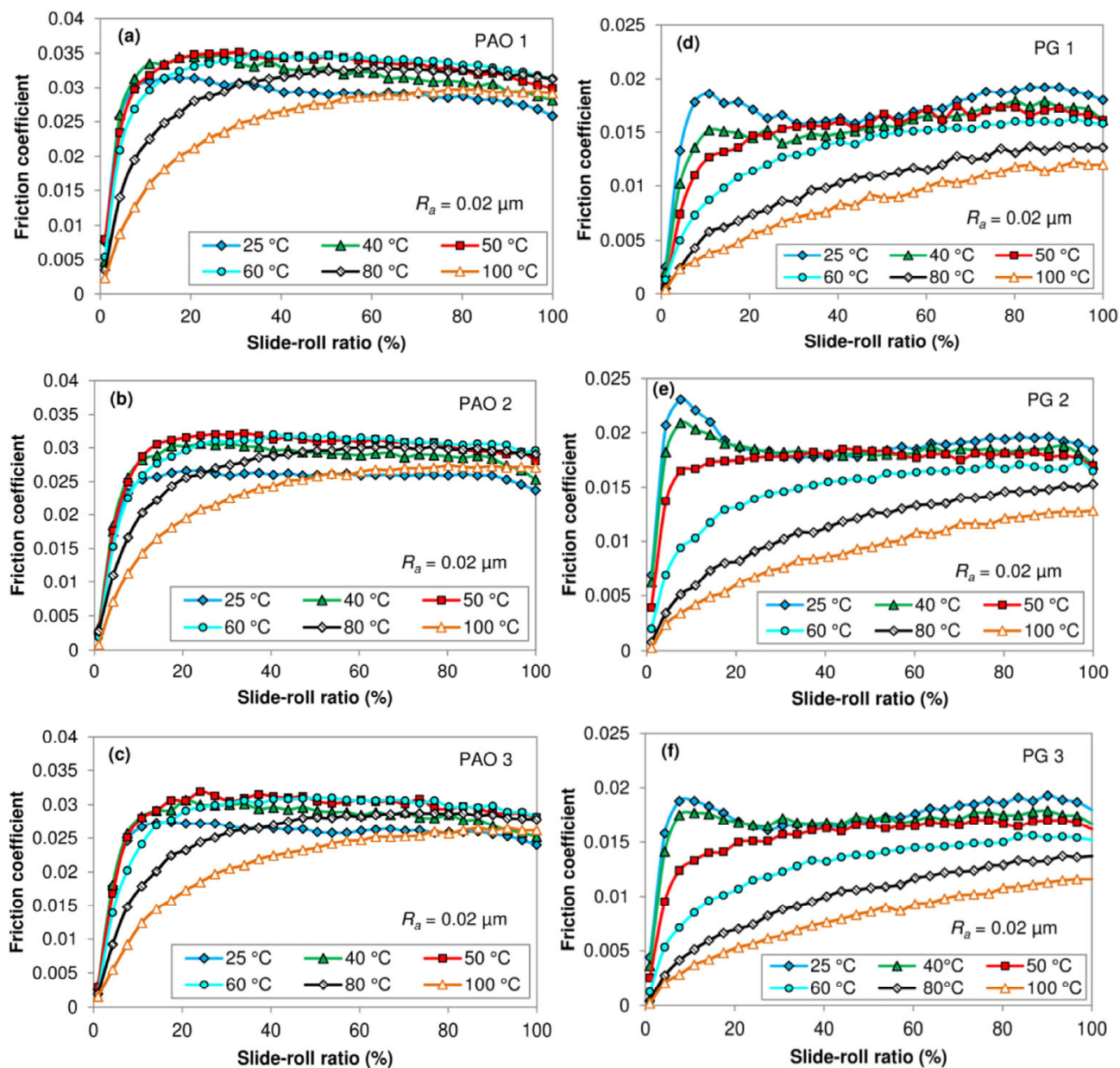

Fig. 7 Traction curves measured for smooth surface $R_{a}=0.02 \mu \mathrm{m}$ with ((a), (b), (c)) PAOs (left), and (d), (e), (f)) PGs (right). Note the vertical scale for PGs is different. 
shown in Fig. 8. Some exceptions include PAO 1 at $40{ }^{\circ} \mathrm{C}$ and PAO 3 at $100{ }^{\circ} \mathrm{C}$. Although patterns described in Fig. 1 is generally valid for EHL condition, it should be noted that traction curves obtained in this study with different roughnesses and temperatures mean that their contacts encountered not only EHL condition but also thin film lubrication condition ( $\lambda$ : between 0.3 and 1), especially at elevated temperatures with rough surfaces. Under all temperature and roughness conditions investigated in this study, the observed friction trend was similar to that commonly found in EHL condition, which is highest friction level with the mineral oil, followed, in order, by PAOs and PGs. This indicates that Fig. 1 is valid for thin film condition too. However, some curves should be carefully interpreted and used. For instance, mineral oil traction curves obtained with $R_{a}=0.2 \mu \mathrm{m}$ at $100{ }^{\circ} \mathrm{C}$ and $R_{a}=$ $0.35 \mu \mathrm{m}$ at $80{ }^{\circ} \mathrm{C}$ and $100{ }^{\circ} \mathrm{C}$ operate in mixed lubrication regime (see Stribeck curve in Fig. 5(c)), so their traction curves are not valid. It is therefore important to verify lubrication regimes from Stribeck curves (Figs. S5 in the ESM) and compare traction curves accordingly. Since the viscosity of PGs at $100{ }^{\circ} \mathrm{C}$ is higher than the mineral oil and PAOs, they form a thicker lubricating film ensuring that their traction curves were obtained
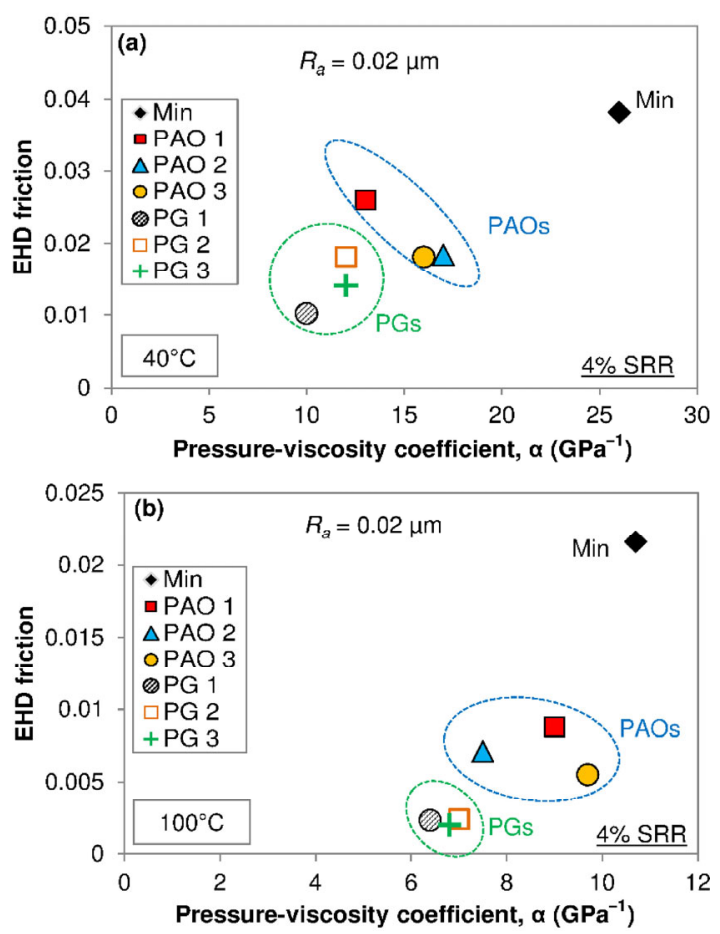

Fig. 8 EHD friction versus pressure-viscosity coefficient for (a) $40{ }^{\circ} \mathrm{C}$ and (b) $100{ }^{\circ} \mathrm{C}$. in thin film/full film condition even with $R_{a}=0.35 \mu \mathrm{m}$ (see Stribeck curves shown in Figs. S5(l), S5(o) and $\mathrm{S} 5(\mathrm{r})$ in the ESM for lubrication regimes).

As can be seen from Fig. S7 in the ESM, regardless of temperature and roughness studied traction curves of PAO 1 display a higher friction level than PAOs 2 and 3. With PGs, while their traction curves with $R_{a}=$ $0.2 \mu \mathrm{m}$ overlap at all temperatures, their curves with $R_{a}=0.35 \mu \mathrm{m}$ generally overlap only up to $50{ }^{\circ} \mathrm{C}$ and curves of PG 1 and 2 deviate to a higher friction level thereafter. Since all three PGs have a similar $\alpha$-value, the observed deviation in friction with PG 1 and 2 might not originate from their $\alpha$-value instead presumably from the way how their molecules respond at high SRRs with $R_{a}=0.35 \mu \mathrm{m}$. Whether or not polymer molecules breakdown at high SRRs, especially in the presence of rough surface $\left(R_{a}=0.35 \mu \mathrm{m}\right)$, remains unclear and needs further investigation but it is apparent that PGs respond differently compared to mineral oil and PAOs at high SRRs.

\section{Discussion}

\subsection{Patterns of traction curves and limiting friction}

Regardless of base oil types, two temperature dependent patterns of behaviour were generally seen with traction curves. One, showing friction increase, levelling out and a drop mainly for low temperatures $\left(25^{\circ} \mathrm{C} \& 40{ }^{\circ} \mathrm{C}\right)$ and the other showing only a friction increase and levelling out for high temperatures. These results suggest that curve types I, II, and III shown in Fig. 1 are applicable to high, medium, and low temperatures, respectively. Similar pattern of behavior was observed with rough surfaces as well (Figs. 6(b) and 6(c)) but due to thinner film and relatively less shear heating type III is relatively less pronounced with rough surfaces, especially at high temperatures where mixed lubrication dominate. By comparing the limiting friction of the seven gear oils tested at the six test temperatures (Fig. 9), it can be seen that mineral oil has higher limiting friction than PAOs and PGs at all temperatures studied. However, the difference in limiting friction between PAOs and PGs is not significant at $25^{\circ} \mathrm{C}$ compared to that observed at higher test temperatures. This is due to reasons described below. 


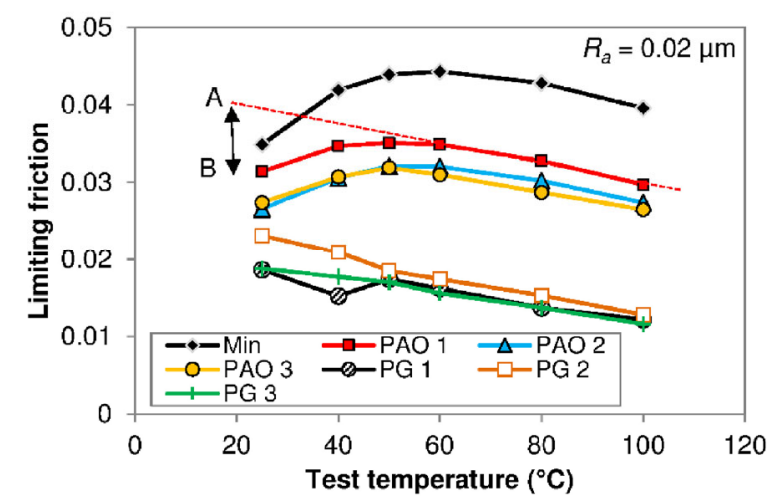

Fig. 9 Limiting friction at different temperatures for all gear oils. Lines are drawn to guide the eyes. The dashed line shown for PAO 1 is the tangent drawn to show the possible limiting friction curve of PAO 1 when no friction drop occurred due to temperature rise. The arrow indicates the difference in limiting friction between case A (without friction drop due to temperature rise) and B (with friction drop due to temperature rise) for PAO 1.

It should be noted that the limiting friction for mineral oil and PAOs slightly increases with the test temperature up to $50{ }^{\circ} \mathrm{C}$ and eventually drops at temperatures above $60{ }^{\circ} \mathrm{C}$ (Fig. 9) while for PGs it always drops with increasing temperature. These results suggest that the measured friction coefficients of mineral oil and PAOs started decreasing due just to temperature rise even before the limiting friction was reached for the test temperatures from $25{ }^{\circ} \mathrm{C}$ to $50{ }^{\circ} \mathrm{C}$ while PGs reached the limiting friction at all temperatures studied. This highlights the importance of correcting the friction for the amount that dropped due to temperature rise to be able to compare the intrinsic friction properties of lubricants. For instance, the response of PAO 1 with friction correction should possibly follow the dashed line shown in Fig. 9 whereas without correction should be like the one shown by the thick line with filled square. The limiting friction at $25{ }^{\circ} \mathrm{C}$ between $\mathrm{A}$ (without friction drop due to temperature rise) and $\mathrm{B}$ (with friction drop due to temperature rise) for PAO 1 differs significantly. Friction comparison without isothermal friction correction may mislead and lead to underestimation of friction properties of lubricants, especially for those that show significant rise in temperature due to shear heating of EHD film. The details of isothermal friction correction made in the present study are discussed in the next section. In general comparing the limiting friction within the PAO family at $60{ }^{\circ} \mathrm{C}$, PAO 3 shows lower limiting friction followed, in order, by PAOs 2 and 1 .
Within the PG family PGs 1 and 3 show slightly lower limiting friction than PG 2.

\subsection{Shear heating of EHD film and isothermal correction of friction}

As described earlier, shear heating of EHD film is predominant with smooth surface and low temperatures. This is associated with the temperature rise in the oil film leading to reduction in friction at high SRRs (Figs. 6(a) and 7). The mean temperature rise of the oil film in the contact, $\Delta \bar{T}$, was estimated using Eq. (1) described in Refs. [14, 18], which is the sum of the mean flash temperature rise of the two surfaces as they pass through the contact $\Delta \bar{T}_{\text {surf }}$ and the mean temperature rise of the oil film above this surface temperature, $\Delta \bar{T}_{\text {oil }}$.

$$
\begin{aligned}
\Delta \bar{T} & =\Delta \bar{T}_{\text {surf }}+\Delta \bar{T}_{\text {oil }} \\
& =\frac{1}{\left(2 \pi K_{s} \rho c\right)^{0.5}}\left(\frac{2 b}{U}\right)^{0.5} \bar{\tau} \Delta U+\frac{h_{c}}{8 K_{\text {oil }}} \bar{\tau} \Delta U
\end{aligned}
$$

where $K_{s}\left(50 \mathrm{~W} / \mathrm{m}^{\circ}{ }^{\circ} \mathrm{C}\right), \rho\left(\sim 8 \mathrm{~g} / \mathrm{cm}^{3}\right)$ and $c \sim 480(\mathrm{~J} / \mathrm{kg} \cdot \mathrm{K})$ are the thermal conductivity, density, and specific heat of the surfaces, respectively, $b$ the half contact width $(0.13 \mathrm{~mm}), K_{\text {oil }}$ the thermal conductivity of the oil film $\left(0.1-0.25 \mathrm{~W} / \mathrm{m} \cdot{ }^{\circ} \mathrm{C}\right), \bar{\tau}$ the mean shear stress over the contact $(=\mu \bar{p}, \mu$ is the measured friction and $\bar{p}$ is the mean pressure), $U$ the entrainment speed, $\Delta U$ the sliding speed, and $h_{\mathrm{c}}$ the central film thickness.

From this the mean oil film temperature in the contact, $\bar{T}_{\text {oil }}$ was calculated using Eq. (2).

$$
\bar{T}_{\text {oil }}=T_{\text {bulk }}+\Delta \bar{T}
$$

where $T_{\text {bulk }}$ is the bulk temperature.

In order to estimate the rate at which the shear stress got reduced for the calculated rise in mean oil film temperature, the mean shear stress and the mean oil film temperature at a fixed strain rate $\left(\dot{\gamma}=\frac{\Delta U}{h_{c}}\right)$ were obtained for all test temperatures, as shown for example in Fig. 10 for mineral oil. A fixed strain rate $\left(3.5 \times 10^{5} \mathrm{~s}^{-1}\right)$ was used mainly to limit the correction to low SRRs as the isothermal correction is reliable only for a temperature rise of $8{ }^{\circ} \mathrm{C}$, which corresponds to about 20\%-30\% SRR at which friction drop occurred. Table 2 lists the strain rates at which friction drop 


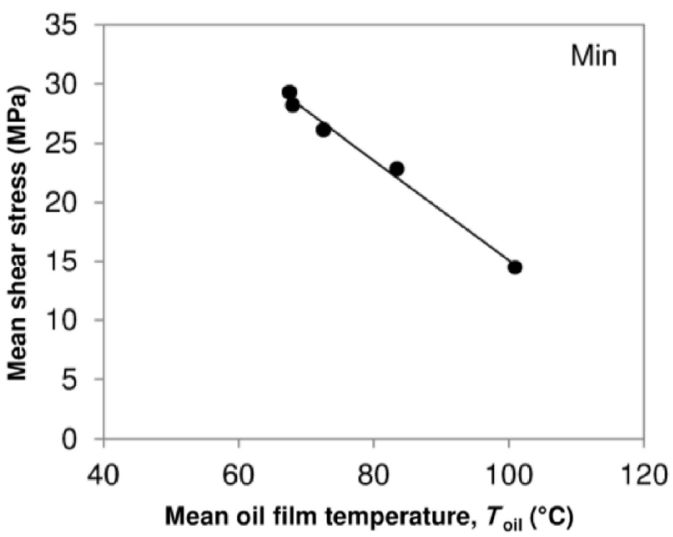

Fig. 10 Mean shear stress versus corrected mean oil film temperature for mineral oil at a fixed strain rate of $3.5 \times 10^{5} \mathrm{~s}^{-1}$.

Table 2 Values of strain rate, mean shear stress, lambda ratio, and calculated mean temperature rise of the oil film in the contact at which friction starts to drop for the smooth surface at $40{ }^{\circ} \mathrm{C}$.

\begin{tabular}{cccc}
\hline Oil & $\begin{array}{c}\text { Strain rate } \\
\left(10^{5} \mathrm{~s}^{-1}\right)\end{array}$ & $\begin{array}{c}\text { Mean shear } \\
\text { stress } \\
(\mathrm{MPa})\end{array}$ & $\begin{array}{c}\text { Calculated mean temperature } \\
\text { rise of the oil film in the } \\
\text { contact, } \Delta T\left({ }^{\circ} \mathrm{C}\right)\end{array}$ \\
\hline Min & 3 & 43 & 6 \\
PAOs & $3.2-3.8$ & $32-36$ & $3.5-4.5$ \\
PGs & $3.2-4.2$ & $22-24$ & $2-3$ \\
\hline
\end{tabular}

occurred for each oil type at the test temperature $40{ }^{\circ} \mathrm{C}$ with the smooth surface. It can be seen for most fluids that friction drop occurred at a strain rate of about $3 \times 10^{5} \mathrm{~s}^{-1}-4 \times 10^{5} \mathrm{~s}^{-1}$ so a mean value of $3.5 \times$ $10^{5} \mathrm{~s}^{-1}$ was used. The associated mean shear stress and mean temperature rise of the oil film in the contact for the identical strain rate is controlled primarily by the oil type, i.e. mineral oil shows higher shear stress and temperature rise followed by PAOs and PGs. The measured film thickness at $2.5 \mathrm{~m} / \mathrm{s}$ was adjusted to contacts in friction tests by a factor of 0.97 (as described in section 4.2) and used for strain rate calculation. The obtained shear stress at each temperature was then added to the measured mean shear stress for isothermal correction. The corrected mean shear stress divided by the mean pressure gives the requisite isothermally corrected friction.

The isothermally corrected traction curves for the mineral oil at different temperatures and for all oils at $40{ }^{\circ} \mathrm{C}$ are shown in Figs. 11(a) and 11(b), respectively. The linear dependence of isothermally corrected friction on SRR is apparent for mineral oil and PAOs, indicating that shear stress varies linearly with strain
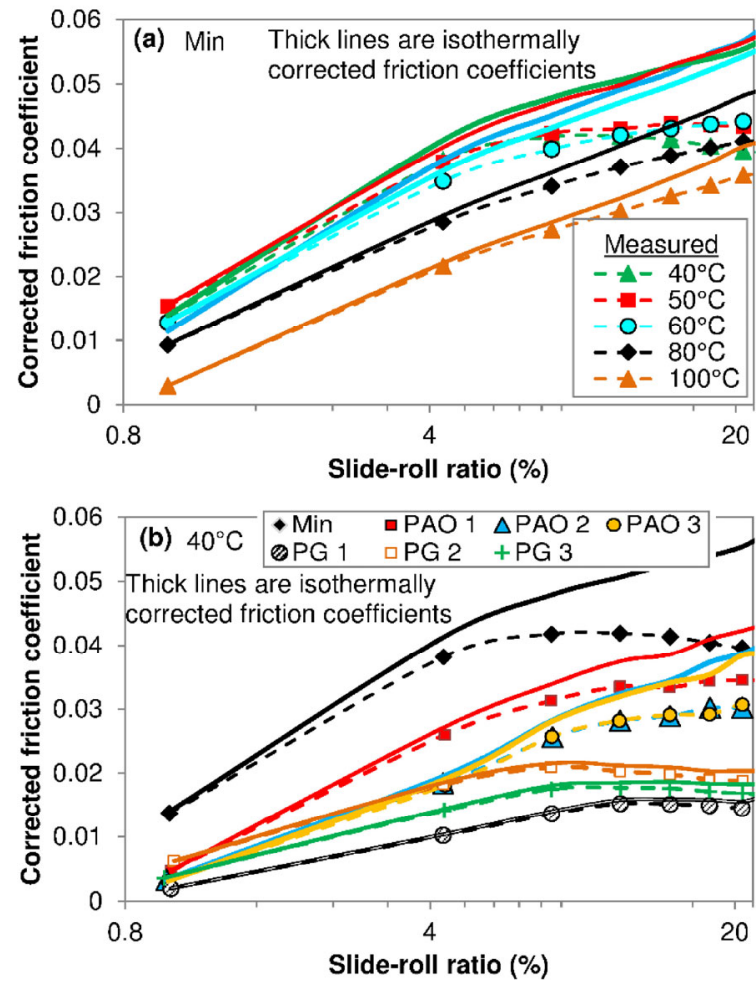

Fig. 11 Isothermally corrected traction curves for (a) mineral oil at different temperatures, and (b) all oils at $40{ }^{\circ} \mathrm{C}$.

rate, especially at low SRRs. However, PGs still show a drop in friction even after isothermal correction, similar to that reported by Zhang et al. in Ref. [18] for one of the PGs they studied. This was attributed to breaking down of polymer molecules but needs further investigation. It should be noted, as widely accepted, that the whole of this correction is reliable only for a maximum temperature rise of $8{ }^{\circ} \mathrm{C}$. This means the corresponding SRR must be very low (less than $5 \%$ for the viscosity grade studied in this work). However like suggested by Zhang et al. in Ref. [18], the linearity continued up to $50 \%$ SRR (although plots are shown only up to $20 \%$ SRR) for mineral oil and POAs, indicating that the correction is valid to a much higher temperature as well.

It is believed that some synthetic oils could be better differentiated by isothermally corrected friction coefficients at moderate SRRs compared to those without any correction. For instance, PAO 2, which at about $60 \%$ SRR, showed the highest corrected friction (not shown here) followed by PAOs 1 and 3. This oil showed lower friction than PAO 1 when measured (uncorrected) friction was used. 


\subsection{Micro-EHD film}

Measuring friction at different temperatures and roughnesses enables constructing a full Stribeck curve covering an extended boundary, mixed, and EHD lubrication regimes. Friction curves obtained at medium to high temperatures $\left(60{ }^{\circ} \mathrm{C}-100{ }^{\circ} \mathrm{C}\right)$ with smooth and rough surfaces merge well and reflect this. This can be seen in Figs. 12, 13, and 14 for mineral oil, PAO 3, and PG 1, respectively, representing one from each base oil type, with three roughnesses. Friction curves of other gear oils are shown in Fig. S4 in the ESM. The curves at $25{ }^{\circ} \mathrm{C}$ with smooth surface show mainly EHL, while with rough surfaces (especially $R_{a}=0.2 \mu \mathrm{m}$ ) show boundary/mixed and just merely the minimum friction of EHL region. With increase in temperature curves shift towards left side, meaning that mixed/ boundary regions build up.

Interestingly, Stribeck curves with $R_{a}=0.2 \mu \mathrm{m}$ show a peculiar bulge (increase in friction) at medium lambda ratios, especially for $40{ }^{\circ} \mathrm{C}-60{ }^{\circ} \mathrm{C}$. This is indicated by arrows in Fig. 12(b). A typical Stribeck curve without such a bulge would generally follow the dashed line (a tangent drawn along the friction curve of $R_{a}=0.2 \mu \mathrm{m}$ ) shown in Fig. 12(b). This means that Stribeck curves with $R_{a}=0.2 \mu \mathrm{m}$ yield a slightly higher friction compared to the typical Stribeck curve without such a bulge. This roughness effect on friction will hereafter be referred to as "a bulge" or "an increase in friction". Such a bulge was seen mainly with mineral oil and PAOs, and was slightly more pronounced with PAOs (indicated by thick upward arrows for $\lambda=2$ in Figs. 12(b) and 13(b)). The increase in friction was found in the lambda ratio range of $0.5-3.5$ (for all oils), where separation of asperities is only partial. This can be seen much clearly from the curves of $40{ }^{\circ} \mathrm{C}, 50{ }^{\circ} \mathrm{C}$, and $60{ }^{\circ} \mathrm{C}$ shown in Fig. S5(b), S5(e) and S5(h) in the ESM. A similar lambda range was reported by Guegan
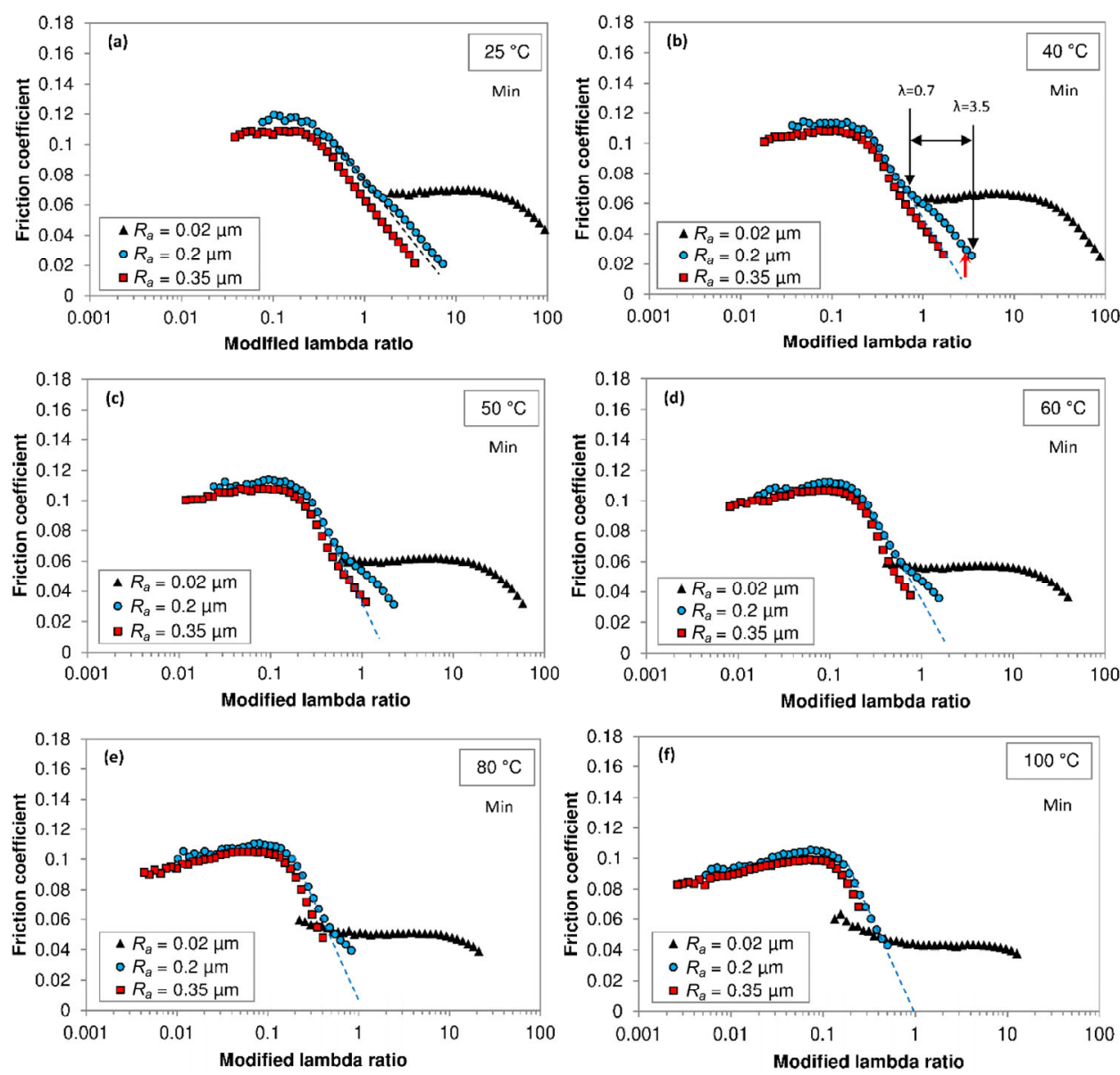

Fig. 12 Stribeck curves of mineral oil at different temperature for three roughnesses. Arrows in (b) indicate friction increase due to micro-EHD formation. 

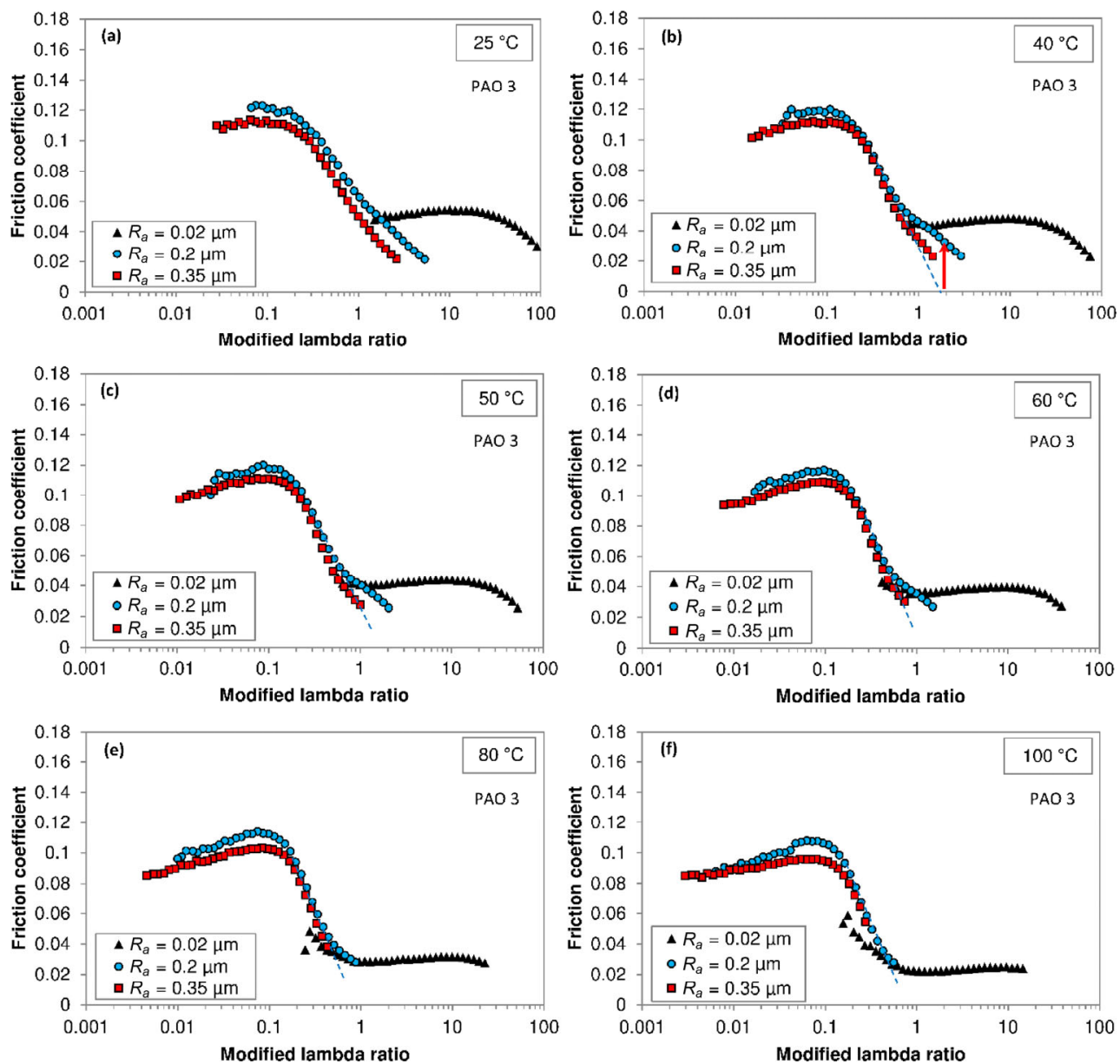

Fig. 13 Stribeck curves of PAO 3 at different temperature for three roughnesses. Arrow shown in (b) indicates friction increase due to micro-EHD formation.

et al. in Ref. [3]. The observed bulge (increase in friction) could be associated with the formation of micro-EHD film and the resulting high shear stress at the top of asperities, as reported by previous authors $[3,6,7,21]$. Such an effect is limited only in the above mentioned range of lambda ratio as under partial separation pressure at the asperity peaks and shear rate are significantly high enough to increase both viscosity and shear stress of the lubricant, hence friction increases. The roughness effect on friction becomes negligible at high lambda ratios, thus macro-EHD film prevails. The evolution from micro- to macro-EHD film can be seen from the curves obtained at different roughnesses as shown in Fig. S4 in the ESM. Curves obtained with $R_{a}=0.02 \mu \mathrm{m}$ show macro-EHD while those particularly in the temperature range $40{ }^{\circ} \mathrm{C}-60{ }^{\circ} \mathrm{C}$ (Figs. S4(b), S4(c), S4(d), S4(h), S4(i), and S4(j) in the $\mathrm{ESM}$ ) with $R_{a}=0.2 \mu \mathrm{m}$ show micro-EHD film formation at high lambda ratios, and to some extent with $R_{a}=$ $0.35 \mu \mathrm{m}$ as well.

The roughness effect on friction can further be seen by plotting $\%$ change in friction alongside the friction coefficient for example for PAO 3, as shown in Fig. 15(a) where the rapid increase in friction in the micro-EHD region is evident. The \% change in friction was obtained by taking \% change between friction measurements made at each consecutive speed. For instance, $\%$ friction $=((\mu 1-\mu 2) / \mu 1) \times 100$, where $\mu 1$ and $\mu 2$ are friction coefficients measured at consecutive speeds 1 and 2, respectively. The \% change in friction curve was smooth fitted by $6^{\text {th }}$ order polynomial equation. An example for conventional EHL or macroEHD is shown in Fig. 15(b) for a $6 \mathrm{~mm}^{2} / \mathrm{s} \mathrm{PAO}$ oil (PAO6 at $100{ }^{\circ} \mathrm{C}$ ) tested at $100{ }^{\circ} \mathrm{C}$ with smooth surface where the observed obvious increase in friction beyond the EHL minima is due to the well described $\sin h^{-1}$ 

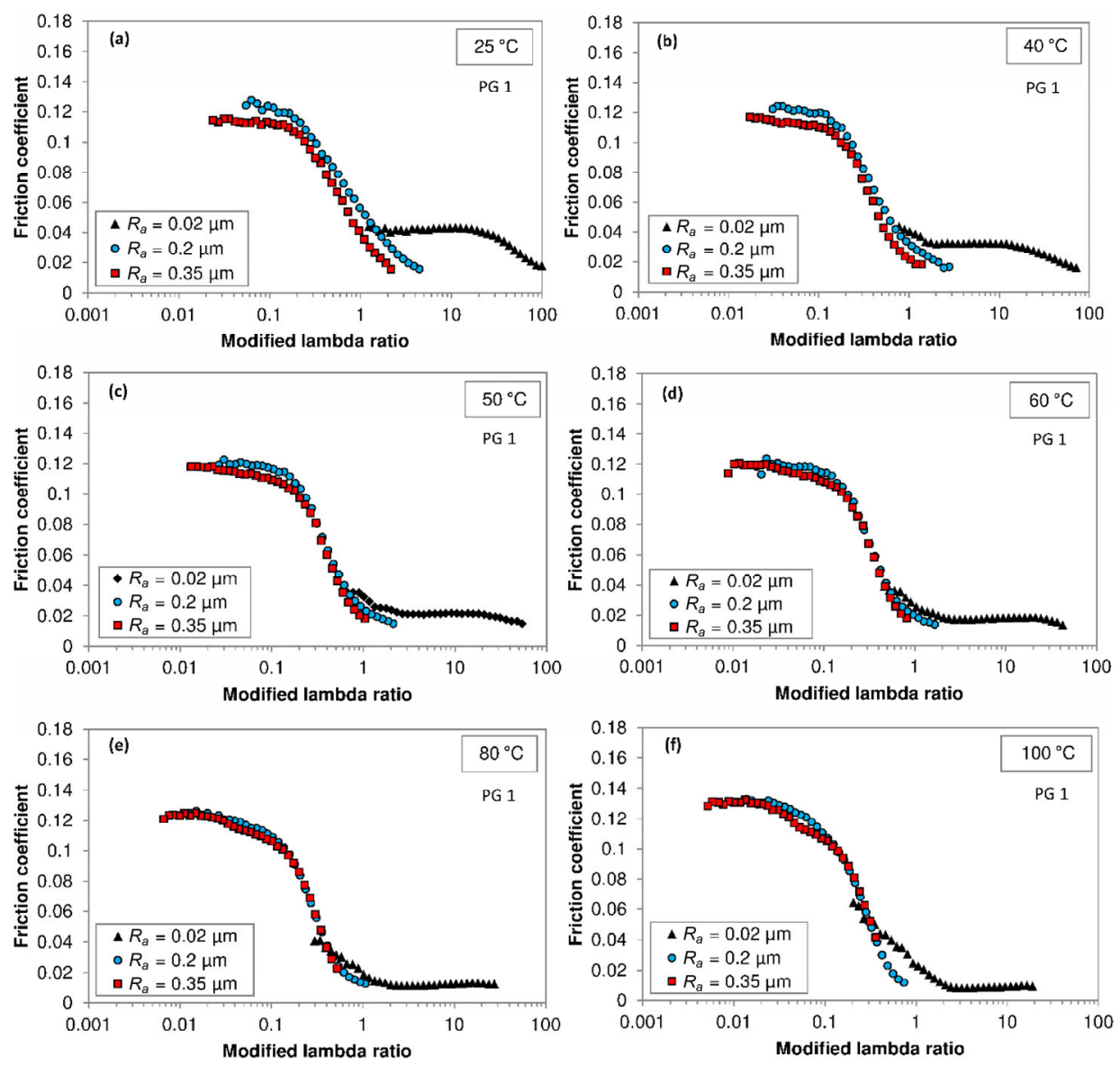

Fig. 14 Stribeck curves of PG 1 at different temperature for three roughnesses.

relationship between shear rate and shear stress. For the conventional macro-EHD case the \% change in friction increases rapidly and normally goes to positive. In addition, a separate test with PAO 3 was conducted with $0.2 \mu \mathrm{m}$ roughness specimen in which an initial Stribeck curve was taken at $40{ }^{\circ} \mathrm{C}$ followed by a prolonged rubbing under boundary lubrication condition $\left(80{ }^{\circ} \mathrm{C}, 0.05 \mathrm{~m} / \mathrm{s}, 50 \% \mathrm{SRR}\right)$ for $10 \mathrm{~h}$ and then a final Stribeck curve at $40{ }^{\circ} \mathrm{C}$ was taken. By comparing the $\%$ change in friction for the curves obtained initially and after $10 \mathrm{~h}$ shown in Fig. 15(c), it can be seen that the initially observed micro-EHD region is slightly suppressed and shifted towards higher lambda ratios after $10 \mathrm{~h}$ due to reduction in roughness $\left(R_{a}=\right.$ $0.08 \mu \mathrm{m}-0.1 \mu \mathrm{m}$ after $10 \mathrm{~h}$ test) over prolonged rubbing. It should be noted in this case that the increase in friction occurs at $\lambda \geq 3$, indicating that the effect of roughness persist even after full separation of the contact. These results further emphasize that the observed increase in friction originates solely from the roughness effect.

The above described increase in friction was observed mainly with mineral oil and PAOs but not with PGs. One possibility could be that polymeric molecules in PGs break down as they pass through the EHD contact leading to reduction in friction [18]. This could be much pronounced with rough surfaces as the shear rate due to thinner film at the asperity peaks is much higher. Alternatively, PGs may have dissipated the generated heat from the micro-EHD film much faster than mineral oil and PAOs due to their higher thermal conductivity. The latter however is unlikely as the calculated temperature rise due to micro-EHD formation was less than $5{ }^{\circ} \mathrm{C}$ for $\lambda$ between 1 and 2 (calculation was made for mineral oil). All these results tend to suggest that friction curves of PGs by-pass the micro-EHD region and follow the classical EHL theory even for rough surfaces (see also Fig. 14 and Figs. S4(m)-S4(x) in the ESM), as shown schematically in Fig. 16. It could be possible, depending on the 

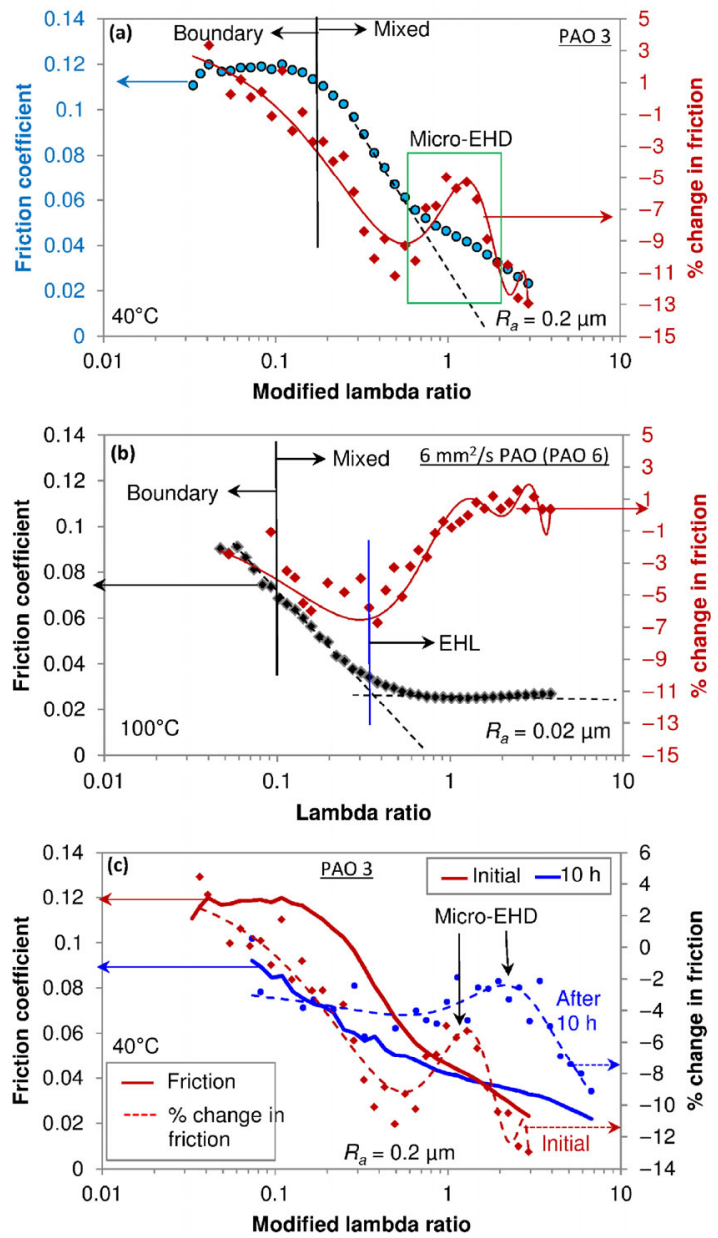

Fig. $15 \%$ change in friction versus modified lambda ratio curves alongside the friction curves for (a) PAO 3 at $40{ }^{\circ} \mathrm{C}$, (b) $6 \mathrm{~mm}^{2} / \mathrm{s}$ PAO oil (PAO 6 at $100{ }^{\circ} \mathrm{C}$ ), and (c) PAO 3 at $40{ }^{\circ} \mathrm{C}$ obtained initially and after $10 \mathrm{~h}$ of rubbing under boundary lubrication condition.

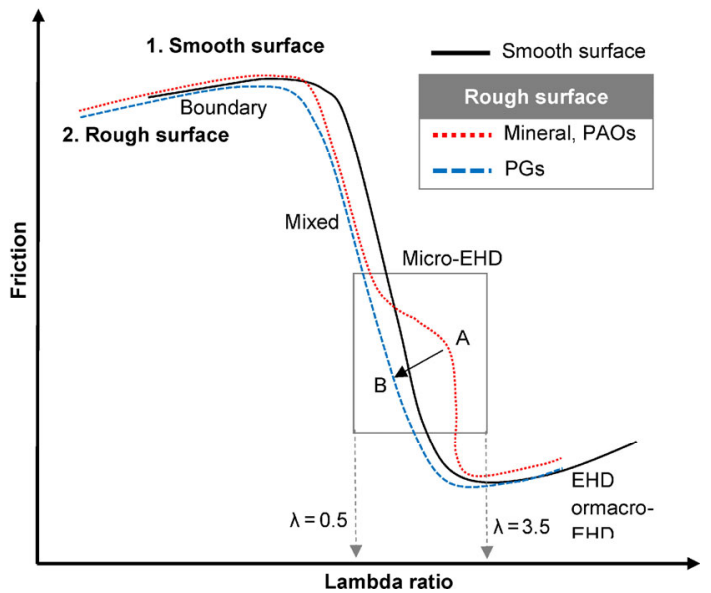

Fig. 16 Schematic diagram showing Stribeck curve shapes in (1) smooth surface with macro-EHD region and low viscosity oils (not those investigated in this study), and (2) rough surface with micro-EHD region and high viscosity oil investigated in this study. Curve shapes in boundary lubrication regime are arbitrary. molecular weight or shear stability, that PGs may behave either like $\mathrm{A}$ or $\mathrm{B}$ in the micro-EHD region shown in Fig. 16. The results from this study suggest that the observed increase in friction with the rough surface (for the lambda between 0.5 and 3.5) results from the combined effect of both viscosity increase due to higher pressure and thinner films due to higher shear rate at the asperity peaks. The possible origin of EHD friction and conditions sensitive to micro-EHD region formation are summarised in Table 3.

\subsection{Practical implications}

In reality, many engineering components like gears initially have rough surfaces which after run-in may attain a roughness lower than the initial but need not necessarily become smooth, meaning that the contacts operate mainly in mixed lubrication. But the roughness after run-in would generally be a contribution from the roughness of additive-derived tribofilm based on its morphology. The effect of roughness from tribofilm on friction is the subject of future study. It is anyway likely that such contacts with medium roughness encounter higher friction by the formation of microEHD film, and this friction can be influenced to some extent by lubricant types like shown by results in this study. It is however not clear and need further investigation on whether this local increase in lubricant viscosity would cause less wear compared to the case without such a micro-EHD film.

\section{Conclusions}

This paper has compared the EHD friction properties of seven ISO VG 320 gear oils covering three main base oil types, namely mineral, PAO, and PG, with three different roughnesses and six temperatures in a lubricated rolling/sliding point contact. Some results from this study confirm the previous work. For the conditions studied in this work the following conclusions can be derived.

(1) Film thickness results indicate that some differences in film thickness within family of similar fluids exist. This is believed to be due to differences in pressure-viscosity coefficient. Film thickness results are in agreement with previous work that it is primarily controlled by pressure-viscosity coefficient 
Table 3 Origin of EHD friction as a function of film thickness, temperature, and roughness.

\begin{tabular}{|c|c|c|c|c|c|}
\hline Nr. & EHD cases & Effects & Friction & Conditions & $\begin{array}{c}\text { Oil type sensitive to } \\
\text { each case }\end{array}$ \\
\hline 1 & Very thick film & Shear heating & Decrease & Smooth surface, low temperature & Mineral, $\mathrm{PAO}, \mathrm{PG}$ \\
\hline 2 & $\begin{array}{l}\text { High localized } \\
\text { pressure due to higher } \\
\text { roughness }\end{array}$ & $\begin{array}{l}\text { Increase in lubricant } \\
\text { viscosity } \& \text { formation of } \\
\text { micro-EHD film }\end{array}$ & Increase & $\begin{array}{c}\text { Rough surface }\left(R_{a}=0.2 \mu \mathrm{m}\right), \\
\text { moderate temperature } \\
\left(40^{\circ} \mathrm{C}-80^{\circ} \mathrm{C}\right), \lambda=0.7-3.5\end{array}$ & Mineral, PAO \\
\hline 3 & $\begin{array}{l}\text { Thin film at the top of } \\
\text { asperities of rougher } \\
\text { surface }\end{array}$ & High shear rate & Increase & $\begin{array}{c}\text { Rough surface }\left(R_{a}=0.2 \mu \mathrm{m}\right), \\
\text { moderate temperature } \\
\left(40^{\circ} \mathrm{C}-80^{\circ} \mathrm{C}\right), \lambda=0.7-3.5\end{array}$ & Mineral, PAO \\
\hline
\end{tabular}

and viscosity.

(2) The results show that the temperature rise of the oil film in the contact can be significantly high with smooth surface at low to moderate temperatures. This could easily result in significant reduction in friction caused by shear heating of the EHD film. It is found that at low test temperatures friction with mineral oil and PAOs starts decreasing (due to temperature rise of the oil film) before reaching the limiting friction while it always reaches the limiting friction with PGs at all test temperatures.

(3) To be able to compare intrinsic friction properties of gear oils without the effects of shear heating and to apply in other contact geometries and condition, it is important to correct friction coefficients for the experienced shear heating. Isothermally corrected friction coefficients could differentiate the fluids of similar types better compared to the uncorrected ones.

(4) The results with rough surfaces show the following: 1) An increase in friction in the lambda range of 0.5-3.5 is observed. This confirms previous work in showing the possibility of micro-EHD film formation at the asperity peaks.

2) The effect of roughness on friction persists even under full film condition $(\lambda \geq 3)$ whereas not at much higher lambda ratios.

3) Mineral oil and PAOs show formation of a micro-EHD region (increased friction at moderate lambda ratios) while PGs do not.

4) Formation of micro-EHD film appears somehow to be temperature-dependent and is found to form only in the temperature range of $40{ }^{\circ} \mathrm{C}-60^{\circ} \mathrm{C}$.

5) Friction curves obtained at different temperatures enable constructing a full Stribeck curve covering an extended range of each lubrication regime. The results reveal that mineral oil and PAOs encounter both micro- and macro-EHD while PGs only macroEHD. It is believed with the PGs investigated in this study that they by-pass the micro-EHD region, possibly by breaking down or shear thinning or by other effects in EHD contact. The molecular weight of fluids may influence the behavior in the lambda range of 0.5 and 3.5 .

\section{Acknowledgements}

The authors wish to thank Mr. Christoph Sedlak for conducting the tribological tests and Dr. Michael Hochmann, Mr. Matthias Pfadt, Mr. Thomas Jorgensen and Dr. Jochen Mühlemeier for organising and performing lubricant analysis. The authors would also like to thank Prof. Hugh Spikes, Imperial College London for discussions and valuable suggestions.

Electronic Supplementary Material: Supplementary material is available in the online version of this article at https://doi.org/10.1007/s12274-019-0267-5.

Open Access: This article is licensed under a Creative Commons Attribution 4.0 International License, which permits use, sharing, adaptation, distribution and reproduction in any medium or format, as long as you give appropriate credit to the original author(s) and the source, provide a link to the Creative Commons licence, and indicate if changes were made.

The images or other third party material in this article are included in the article's Creative Commons licence, unless indicated otherwise in a credit line to the material. If material is not included in the article's Creative Commons licence and your intended use is not permitted by statutory regulation or exceeds the 
permitted use, you will need to obtain permission directly from the copyright holder. To view a copy of this licence, visit http://creativecommons.org/licenses/ by $/ 4.0 \%$.

\section{References}

[1] Greaves M. Pressure viscosity coefficients and traction properties of synthetic lubricants for wind turbine gear systems. Lubr Sci 24(2): 75-83 (2012)

[2] Gunsel S, Korcek S, Smeeth M, Spikes H A. The elastohydrodynamic friction and film forming properties of lubricant base oils. Tribol Trans 42(3): 559-569 (1999)

[3] Guegan J, Kadiric A, Gabelli A, Spikes H A. The relationship between friction and film thickness in EHD point contacts in the presence of longitudinal roughness. Tribol Lett 64(3): 33 (2016)

[4] Vengudusamy B, Grafl A, Novotny-Farkas F, Schöfmann W. Influence of surface roughness on the tribological behavior of gear oils in steel-steel contacts: Part I-Boundary friction properties. Tribol Trans 57(2): 256-266 (2014)

[5] Vengudusamy B, Grafl A, Novotny-Farkas F, Schöfmann W. Influence of surface roughness on the tribological behavior of gear oils in steel-steel contacts: Part II-Mixed friction properties. Tribol Trans 57(4): 561-569 (2014)

[6] Jefferis J A, Johnson K L. First paper: sliding friction between lubricated rollers. Proc Inst Mech Eng 182(1): 281-291 (1967)

[7] Evans C R, Johnson K L. The influence of surface roughness on elastohydrodynamic traction. Proc Inst Mech EngPart $C$ $J$ Mech Eng Sci 201(2): 145-150 (1987)

[8] Britton R D, Elcoate C D, Alanou M P, Evans H P, Snidle $\mathrm{R}$ W. Effect of surface finish on gear tooth friction. $J$ Tribol 122(1): 354-360 (2000)

[9] Jeng Y R. Experimental study of the effects of surface roughness on friction. Tribol Trans 33(3): 402-410 (1990)

[10] Jacod B, Venner C H, Lugt P M. Influence of longitudinal roughness on friction in EHL contacts. $J$ Tribol 126(3): 473-481 (2004)

[11] LaFountain A R, Johnston G J, Spikes H A. The elastohydrodynamic traction of synthetic base oil blends. Tribol Trans 44(4): 648-656 (2001)

[12] Martins R, Seabra J, Brito A, Seyfert C, Luther R, Igartua A. Friction coefficient in FZG gears lubricated with industrial gear oils: biodegradable ester vs. mineral oil. Tribol Int 39(6): 512-521 (2006)

[13] Fernandes C M C G, Martins R C, Seabra J H O. Friction torque of cylindrical roller thrust bearings lubricated with wind turbine gear oils. Tribol Int 59: 121-128 (2013)

[14] Spikes H A, Zhang J. History, origins and prediction of elastohydrodynamic friction. Tribol Lett 56(1): 1-25 (2014)

[15] Hentschel K-H. The influence of molecular structure on the frictional behaviour of lubricating fluids. J Synth Lubr 2(2):
143-165 (1985)

[16] Höhn B R, Michaelis K, Doleschel A. Frictional behaviour of synthetic gear lubricants. Tribol Ser 39: 759-768 (2001)

[17] Muraki M. Molecular structure of synthetic hydrocarbon oils and their rheological properties governing traction characteristics. Tribol Int 20(6): 347-354 (1987)

[18] Zhang J, Tan A, Spikes H. Effect of base oil structure on elastohydrodynamic friction. Tribol Lett 65(1): 13 (2017)

[19] Jacod B, Venner C H, Lugt P M. Extension of the friction mastercurve to limiting shear stress models. J Tribol 125(4): 739-746 (2003)

[20] Martinie L, Vergne P. Tribology in transportation: the contribution of the lubricant rheology for optimizing the behavior of EHD contacts. In First African Congress in Tribology, Marrakech, Morocco, 2014.

[21] Bair S, Winer W O. Regimes of traction in concentrated contact lubrication. J Lubr Technol 104(3): 382-386 (1982)

[22] Nanbu T, Chiba N, Kano M, Ushijima K. Effect of surface roughness on elastohydrodynamic traction: Part 1. Lubr Sci 17(3): 281-293 (2005)

[23] Björling M, Larsson R, Marklund P, Kassfeldt E. Elastohydrodynamic lubrication friction mapping-the influence of lubricant, roughness, speed, and slide-to-roll ratio. Proc Inst Mech Eng Part J: J Eng Tribol 225(7): 671-681 (2011)

[24] Zapletal T, Sperka P, Krupka I, Hartl M. The effect of surface roughness on friction and film thickness in transition from EHL to mixed lubrication. Tribol Int 128: 356-364 (2018)

[25] Venner C H, Lubrecht A A. Numerical analysis of the influence of waviness on the film thickness of a circular EHL contact. J Tribol 118(1): 153-161 (1996)

[26] Lubrecht A A, Venner C H. Elastohydrodynamic lubrication of rough surfaces. Proc Inst Mech Eng Part J: J Eng Tribol 213(5): 397-404 (1999)

[27] Guegan J, Kadiric A, Spikes H. A study of the lubrication of EHL point contact in the presence of longitudinal roughness. Tribol Lett 59(1): 22 (2015)

[28] Greenwood J A, Morales-Espejel G E. The behaviour of transverse roughness in EHL contacts. Proc Inst Mech Eng Part J: J Eng Tribol 208(2): 121-132 (1994)

[29] Johnston G J, Wayte R, Spikes H A. The measurement and study of very thin lubricant films in concentrated contacts. Tribol Trans 34(2): 187-194 (1991)

[30] Spikes H. Basics of EHL for practical application. Lubr Sci 27(1): 45-67 (2015)

[31] Chittenden R J, Dowson D, Dunn J F, Taylor C M, Johnson K L. A theoretical analysis of the isothermal elastohydrodynamic lubrication of concentrated contacts. I. Direction of lubricant entrainment coincident with the major axis of the Hertzian contact ellipse. Proc R Soc Lond Ser A 397(1813): 245-269 (1985)

[32] Vengudusamy B, Enekes C, Spallek R. On the film forming and friction behaviour of greases in rolling/sliding contacts. Tribol Int 129: 323-337 (2019) 


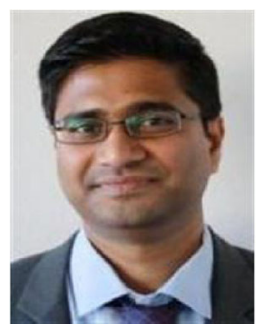

Balasubramaniam VENGUDUSAMY. He graduated in mechanical engineering and obtained his Ph.D in tribology from Imperial College

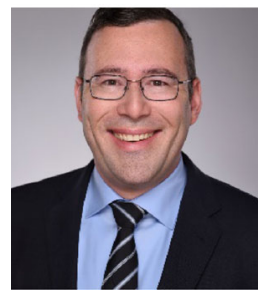

Claus ENEKES. He studied mechanical engineering at RWTH Aachen University, Germany. Following his interest in tribology, he worked in

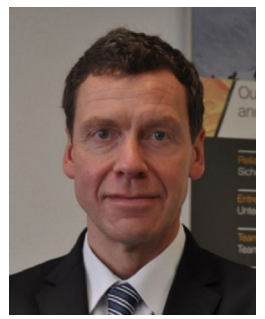

Reiner SPALLEK. He is currently working at Klüber Lubrication München SE \& Co. KG as the manager of tribology section for the Klüber
London, UK. He has been working in the field of tribology for about 15 years. He is currently a senior research tribologist at Klüber Lubrication, Munich.

his Ph.D time on coatings and environmentally friendly lubricants in hydraulic pumps. Since 2012, he is working with Klüber Lubrication München SE \& Co. KG, Germany.

group. He received his master of mechanical engineering at the University of Frankfurt am Main in Germany. He has been working in the field of tribology for over 24 years. 